\title{
Fano fingerprints of Majoranas in Kitaev dimers of superconducting adatoms
}

\author{
F.A. Dessotti ${ }^{a}$, L.S. Ricco ${ }^{a}$, Y. Marques ${ }^{a}$, R.S. Machado ${ }^{a}$, L.H. Guessi ${ }^{b}$, M.S. Figueira ${ }^{c}$, \\ M. de Souza ${ }^{\mathrm{b}}$, A.C. Seridonio ${ }^{\mathrm{a}, \mathrm{b}, *}$ \\ a Departamento de Física e Química, Universidade Estadual Paulista, 15385-000 Ilha Solteira, São Paulo, Brazil \\ ${ }^{\mathrm{b}}$ Instituto de Geociências e Ciências Exatas - IGCE, Universidade Estadual Paulista, Departamento de Física, 13506-970 Rio Claro, São Paulo, Brazil

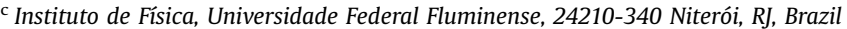

\section{H I G H L I G H T S}

- A multitip device is proposed to probe Majorana fermions in adatoms.

- The transmittance is universal due to the energy swap in the AFM tip.

- Overlapped Majorana fermions lead to a gate voltage invariance feature.

\section{A R T I C L E I N F O}

\section{Article history:}

Received 7 March 2016

Received in revised form 25 April 2016

Accepted 2 May 2016

Available online 3 May 2016

Keywords:

Fano effect

Kitaev dimer

Superconducting adatoms

STM tip

AFM tip

\begin{abstract}
A B S T R A C T
We investigate theoretically a Fano interferometer composed by STM and AFM tips close to a Kitaev dimer of superconducting adatoms, in which the adatom placed under the AFM tip, encloses a pair of Majorana fermions (MFs). For the binding energy $\Delta$ of the Cooper pair delocalized into the adatoms under the tips coincident with the tunneling amplitude $t$ between them, namely $\Delta=t$, we find that only one MF beneath the AFM tip hybridizes with the adatom coupled to the STM tips. As a result, a gate invariance feature emerges: the Fano profile of the transmittance rises as an invariant quantity depending upon the STM tips Fermi energy, due to the symmetric swap in the gate potential of the AFM tip. (c) 2016 Elsevier B.V. All rights reserved.
\end{abstract}

\section{Introduction}

The physicist Ettore Majorana proposed in the field of highenergy Physics the existence of peculiar fermions that constitute their own antiparticles. In the context of condensed matter Physics, these fermions are Majorana quasiparticles [1,2]. From the quantum computing perspective, two Majorana fermions (MFs) can compose a regular fermion acting as a protected qubit, which is indeed decoupled from the host environment and free of the decoherence effect. As a result, the quest for setups supporting MFs has attracted broad interest from the communities of researchers in the fields of quantum information and transport [35], since the qubit made by the coupled MFs appears only in the topological phase. Noteworthy, the Kitaev chain within such a

\footnotetext{
* Corresponding author at: Departamento de Física e Química, Universidade Estadual Paulista, 15385-000 Ilha Solteira, São Paulo, Brazil.

E-mail address: seridonio@dfq.feis.unesp.br (A.C. Seridonio).
}

phase [6] is considered the most promising candidate to this end as the aftermath of the emerging $p$-wave and spinless superconductivity. Indeed, in Kitaev's setup, MFs appear as zero-energy modes attached to the edges of the chain.

Experimentally, $p$-wave superconductivity is feasible due to the proximity effect by the employment of an $s$-wave superconductor close to a semiconducting nanowire characterized by a spin-orbit interaction under an external magnetic field [7-10]. Particularly in the case of transport through quantum dots (QDs) coupled to a MF [11-23], a zero-bias peak (ZBP) $[15,16]$ in the conductance is expected to be observed. It is worth mentioning that the ZBP has been detected in conductance measurements through a nanowire of indium antimonide linked to gold and niobium titanium nitride electrodes [24]. Analogously, a ZBP has also been verified in the superconducting system of aluminium next to a nanowire of indium arsenide [25]. However, the ZBP signature may also have another physical origin, for instance the Kondo effect [26-30], thus turning the experimental ZBP detection inconclusive within a MF perspective. Moreover, recently an alternative way for the achievement of 

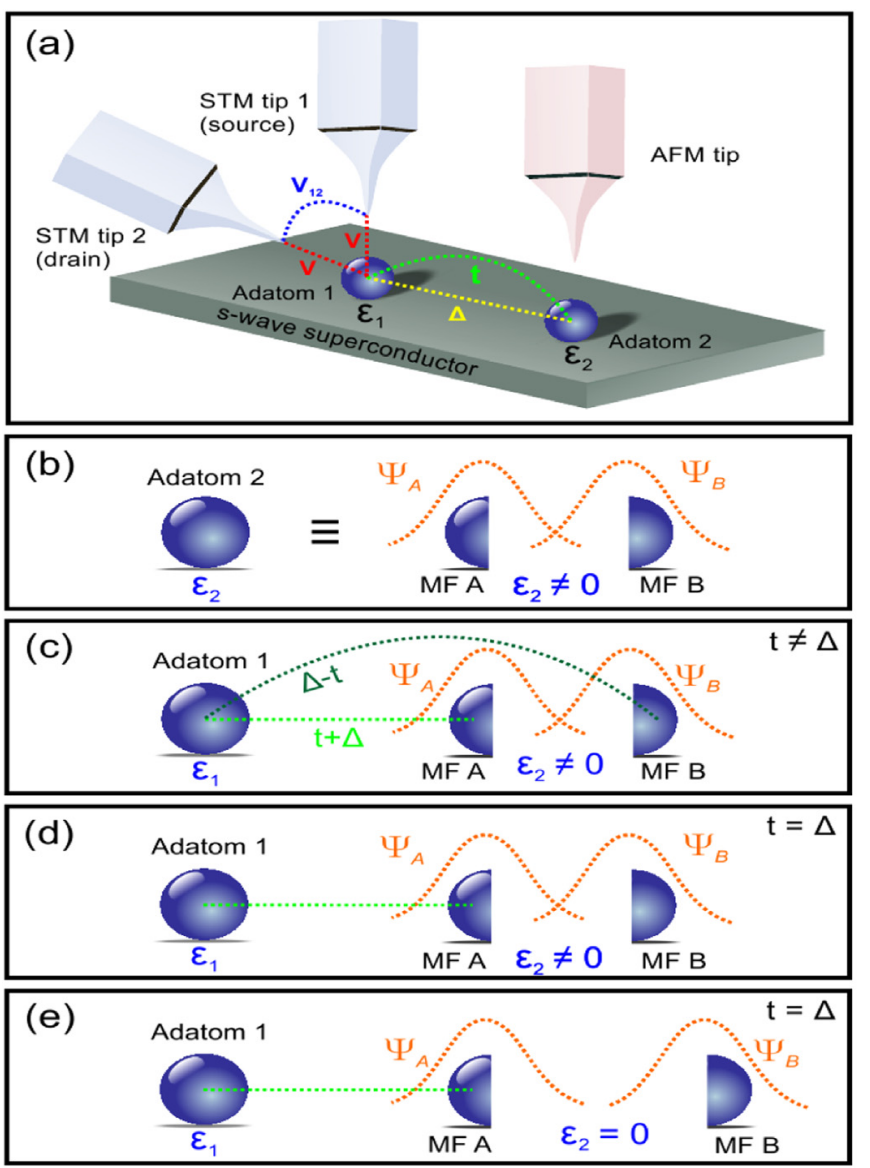

Fig. 1. (a) Setup composed by STM and AFM tips in the presence of superconducting adatoms hosted by a conventional superconductor with strong spinorbit coupling. (b) The adatom 2 is made by a pair of coupled Majorana fermions (MFs $A$ and $B$ represented by half-spheres) where the level $\varepsilon_{2}$ is induced by the AFM tip and plays the role of the connection between the MFs. It can assume negative values when it stays below the MF zero mode, due to the tuning performed by the gate potential of the AFM tip. (c) In the case of $t \neq \Delta$ and $\varepsilon_{2} \neq 0$, both MFs hybridize with the adatom 1. (d) For the matching of the Cooper pair binding energy with the normal tunneling between the adatoms 1 and $2(t=\Delta)$ and $\varepsilon_{2} \neq 0$, the MF $A$ becomes the unique Majorana connected to the adatom 1. (e) Here we see the isolated MF $A$ coupled to the adatom 1 when $t=\Delta$ and $\varepsilon_{2}=0$, which results in the standard $\mathrm{ZBP}$ in the transmittance and in the isolated MF $B$ at one edge of the Kitaev dimer.

the topological Kitaev chain has been the employment of magnetic chains on top of superconductors [31-36]. Particularly in Ref. [34], the ZBP observed exhibits a subtle amplitude of the order $10^{-4}\left(2 G_{0}\right)$, which is a signal extremely weak due to thermal broadening, where $G_{0}=e^{2} / h$ is the quantum of conductance. Thus in the current context, novel approaches in the pursuit of MFs become necessary. In this scenario, we highlight the proposal found in Ref. [37], which is a pioneering example concerning this issue: a hybrid spin-microcantilever system for optical detection of MFs on the edges of a Kitaev chain.

In this work we propose a new route for detection of MFs signatures. To that end, we consider the setup outlined in Fig. 1 where an $s$-wave superconductor with strong spin-orbit coupling hosts a set of magnetic adatoms, in analogy to the experimental apparatus developed in Ref. [34] that describes a superconducting surface of lead $(\mathrm{Pb})$ well known by its strong spin-orbit coupling, thus allowing $p$-wave superconductivity on this chain due to the proximity effect. It is worth mentioning that the proposal of Ref. [34] is distinct from the semiconducting spin-orbit wire reported in Ref. [24]. In the current work, we consider for the sake of simplicity, just a pair of superconducting adatoms (Kitaev dimer), in particular placed nearby STM (Scanning Tunneling Microscope) and AFM (Atomic Force Microscope) tips. Additionally, we assume two highly spin-polarized STM tips in order to freeze the spin degree of freedom, thus avoiding the emergence of the Kondo effect within the adatom 1.

In regard to the possibility of experimental realization of the setup shown in Fig. 1, we stress that multitip STM experiments can be possible, see e.g. Refs. [38-40]. In the case of the AFM tip, which is fixed on top of an adatom, it operates similarly to the Scanning Gate Microscopy (SGM) technique [41], wherein a charged tip allows that its gate potential tunes the energy levels of the adatom probed. This approach is widely employed in systems with quantum point contacts (QPCs) formed in two dimensional electron gases $[42,43]$, which we invoke here to our proposal. Thereby despite the challenging of applying the SGM technique in the scenario of Fig. 1, we trust that in the near future such a procedure can be implemented.

In the frame of the setup here proposed, a device based on Fano interference $[44,45,21,22]$ becomes an alternative method for detection of MFs. As a matter of fact, the Fano effect is found in systems where distinct tunneling paths compete for the electron transport. Noteworthy, such a phenomenon can be perceived experimentally by the STM just by measuring the conductance [2630]. In the setup of Fig. 1 here proposed, the Fano effect arises from the adatom 1 coupled simultaneously to the pair of STM tips, once the electrons can tunnel between such tips or (and) directly through this adatom as expected for realistic experimental conditions. Thus depending on the Fano lineshape of the conductance, we can identify which path rules the quantum transport or if they compete on the same footing. Later on, we will see that the Fano parameter of interference $q_{b}$ then dictates the Fano profile making explicit the dominant path in the system.

Here we show that the two coupled MFs $A$ and $B$ within the adatom under the AFM tip (see Fig. 1) lead to a gate invariance feature in the transport experiment. Particularly, when the AFM tip swaps symmetrically its gate potential around the MF zero mode, it reveals a universality signature in the Fano profile of the transmittance. More specifically, we find then distinct situations in which the transmittance shares the same profile as a function of the Fermi level of the STM tips, in particular when the binding energy of the Cooper pair splits into the adatoms under the STM and AFM tips, is in resonance with the tunneling term between them. In this case, we show that when the MF A couples solely to the adatom 1 , the gate invariance characterized by the aforementioned universality is revealed by connecting MFs $A$ and $B$.

This paper is organized as follows: in Section 2 we develop the theoretical model for the system sketched in Fig. 1 by deriving the expression for the transmittance through such a device (see also the Appendix) together with the system Green's functions. The results are presented in Section 3, and in Section 4 we summarize our concluding remarks.

\section{The model}

\subsection{System Hamiltonian}

Here we consider the system outlined in Fig. 1(a) for a pair of highly spin-polarized STM tips connected to the hybrid setup composed by an AFM tip and the "host+superconducting adatoms" set. Thus such a spinless model reads:

$$
\begin{aligned}
\mathcal{H}= & \sum_{\alpha k} \tilde{\varepsilon}_{\alpha k} c_{\alpha k}^{\dagger} c_{\alpha k}+\sum_{j} \varepsilon_{j} d_{j}^{\dagger} d_{j}+V \sum_{\alpha k}\left(c_{\alpha k}^{\dagger} d_{1}+\text { H.c. }\right) \\
& +\left(V_{12} \sum_{k q} c_{1 k}^{\dagger} c_{2 q}+t d_{1} d_{2}^{\dagger}+\Delta d_{2}^{\dagger} d_{1}^{\dagger}+\text { H.c. }\right),
\end{aligned}
$$


where the electrons in the STM tip $\alpha=1,2$ (STM tips 1 and 2, respectively) are described by the operator $c_{\alpha k}^{\dagger}\left(c_{\alpha k}\right)$ for the creation (annihilation) of an electron in a quantum state labeled by the wave number $k$ and energy $\tilde{\varepsilon}_{\alpha k}=\varepsilon_{k}-\mu_{\alpha}$, with $\mu_{\alpha}$ being the chemical potential. Here we adopt the gauge $\mu_{2}=\Delta \mu$ and $\mu_{1}=-\Delta \mu$, with $\mu_{2}-\mu_{1}=2 \Delta \mu=e \varphi$ being the bias between the tips, being $e>0$ the electron charge and $\varphi$ the bias-voltage. Consequently, the transmittance through the setup is a function of the Fermi energy $\varepsilon=\mu_{1}=\mu_{2}$ of the STM tips, where the point $\varepsilon=0$ corresponds to the MF zero mode. For the adatoms, $d_{j}^{\dagger}\left(d_{j}\right)$ creates (annihilates) an electron in the state $\epsilon_{\mathrm{j}}$, with $j=1,2 . V$ stands for the hybridizations between the adatom 1 and the STM tips. These couplings are considered the same to avoid Andreev currents in the system $[11,12]$ (see also the Appendix). $V_{12}$ is the STM tip 1-tip 2 coupling, which will ensure the precise renormalization of the energy level in the adatom 1 as we will see.

The $s$-wave superconductor with strong spin-orbit coupling enters into the model via the parameters $t$ and $\Delta$, which respectively yield the hopping term between the adatoms 1 and 2, and also the binding energy of a delocalized Cooper pair split into such adatoms: as a net effect of the arrangement of Fig. 1(a) considered, $p$-wave superconductivity is induced on the pair of adatoms (Kitaev dimer) close to the tips, similarly to that reported in Ref. [34] for the case of a long Kitaev chain. As the adatom 1 is placed within the region separating the STM tips and the superconducting surface, we can safely neglect the tunneling from the former into the latter as an outcome of the setup adopted. We stress that the role of the AFM tip is indeed to gate overlap the MFs enclosed by the adatom 2 . This clue between the MFs found within such an adatom will be clarified later on as the own energy level of this adatom, which consists in the degree of freedom completely tunable by the AFM tip. Thus for the sake of simplicity, we clamped these features as the most relevant from the proximity problem between the $s$-wave surface and the adatoms. Alternatively to this end, an approach concerning $a b$ initio description on the proximity issue can be helpful, but it does not belong to the current scope of the paper.

Here we express the adatom 2 in the Majorana basis by following the transformation $d_{2}=\frac{1}{\sqrt{2}}\left(\Psi_{A}+i \Psi_{B}\right)$ and $d_{2}^{\dagger}=\frac{1}{\sqrt{2}}\left(\Psi_{A}-i \Psi_{B}\right)$, in which $\Psi_{l}^{\dagger}=\Psi_{l} \quad(l=A, B)$ characterizes a MF operator, thus yielding

$$
\begin{aligned}
\mathcal{H}_{\mathrm{MFs}} & =\varepsilon_{2} d_{2}^{\dagger} d_{2}+\left(t d_{1} d_{2}^{\dagger}+\Delta d_{2}^{\dagger} d_{1}^{\dagger}+\text { H.c. }\right) \\
& =i \varepsilon_{2} \Psi_{A} \Psi_{B}+\frac{(t+\Delta)}{\sqrt{2}}\left(d_{1}-d_{1}^{\dagger}\right) \Psi_{A}+i \frac{(\Delta-t)}{\sqrt{2}}\left(d_{1}+d_{1}^{\dagger}\right) \Psi_{B}+\frac{\varepsilon_{2}}{2},
\end{aligned}
$$

where the first term in the second line makes explicit the MFs within the adatom 2 and the others stand for the connections between MFs $A$ and $B$ with the adatom 1 (see Fig. 1(c)-(e)). It is worth noticing that the standard Majorana ZBP occurs for $t=\Delta, \varepsilon_{1} \neq 0$ and $\varepsilon_{2}=0$, with the conductance $G=0.5 G_{0}$ due to the MF $\gamma_{1}=i\left(d_{1}-d_{1}^{\dagger}\right) / \sqrt{2}$ existing in the adatom 1 given by the second term of Eq. (2) (-2it $\left.\gamma_{1} \Psi_{A}\right)$, which has a component on the adatom 2 , where a completely localized MF is observed according to Leijnse and Flensberg in Ref. [5].

In this scenario, we explore the model regimes $t=\Delta$ and $t \neq \Delta$ combined with $\varepsilon_{2}>0$ and $\varepsilon_{2}<0$ varied symmetrically with respect to the MF zero mode. We stress that for a fixed $\Delta$, the tuning of the parameter $t$ can be performed experimentally just by changing the distance between the adatoms 1 and 2, while the AFM tip controls the level position $\epsilon_{2}$ of the adatom 2, since such a tip operates as a gate potential. Particularly for $t=\Delta$, we can verify in Eq. (2) that the adatom 1 decouples from the MF $B$ (here sketched by the most right half-sphere in Fig. 1(d)) thus allowing an exclusively connection between this adatom and the MF $A$ (the most left half-sphere) as the Hamiltonian $\mathcal{H}_{\mathrm{MFs}}=\varepsilon_{2}\left(i \Psi_{A} \Psi_{B}+\frac{1}{2}\right)+\sqrt{2} \Delta\left(d_{1}-d_{1}^{\dagger}\right) \Psi_{A}$ points out.
For $\varepsilon_{2} \neq 0$, the Fano profile of the transmittance becomes invariant under the symmetric change of $\varepsilon_{2}$ with respect to the MF zero mode, in contrast with the case $t \neq \Delta$ where this universality is prevented. Thereafter, it gives rise to distinct transmittance profiles strongly dependent on the sign of $\varepsilon_{2}$ away from the point $t=\Delta$.

Thereby we recognize this invariance as a Majorana property of the system when it is driven to the point $t=\Delta$ with symmetric swap of $\varepsilon_{2}$.

\subsection{Conductance}

In what follows we derive the Landauer-Büttiker formula for the zero-bias conductance $G$ [46]. Such a quantity is a function of the transmittance $\mathcal{T}(\varepsilon)$ as follows:

$G=G_{0} \int d \varepsilon\left(-\frac{\partial f_{F}}{\partial \varepsilon}\right) \mathcal{T}(\varepsilon)$

where $f_{F}$ stands for the Fermi-Dirac distribution.

We begin with the transformations $c_{2 k}=\frac{1}{\sqrt{2}}\left(c_{e k}+c_{o k}\right)$ and $c_{1 k}=\frac{1}{\sqrt{2}}\left(c_{e k}-c_{o k}\right)$ on the Hamiltonian of Eq. (1), which starts to depend on the even and odd conduction operators $c_{e k}$ and $c_{o k}$, respectively. These definitions allow us to express Eq. (1) as $\mathcal{H}=\mathcal{H}_{e}+\mathcal{H}_{o}+\tilde{\mathcal{H}}_{\text {tun }}=\mathcal{H}_{\varphi=0}+\tilde{\mathcal{H}}_{\text {tun }}$, where

$$
\begin{aligned}
\mathcal{H}_{e}= & \sum_{k} \varepsilon_{k} c_{e k}^{\dagger} c_{e k}+\varepsilon_{1} d_{1}^{\dagger} d_{1}+V_{12} \sum_{k p} c_{e k}^{\dagger} c_{e p}+\sqrt{2} V \sum_{k}\left(c_{e k}^{\dagger} d_{1}+\text { H.c. }\right) \\
& +\mathcal{H}_{\mathrm{MFs}}
\end{aligned}
$$

represents the Hamiltonian part of the system coupled to the adatoms via an effective hybridization $\sqrt{2} V$, while

$\mathcal{H}_{o}=\sum_{k} \varepsilon_{k} c_{o k}^{\dagger} c_{o k}-V_{12} \sum_{k p} c_{o k}^{\dagger} c_{o p}$

is the decoupled one. However, they are connected to each other by the tunneling Hamiltonian $\tilde{\mathcal{H}}_{\text {tun }}=-\Delta \mu \sum_{k}\left(c_{e k}^{\dagger} c_{o k}+c_{o k}^{\dagger} c_{e k}\right)$.

As in the zero-bias regime $\Delta \mu \rightarrow 0$, due to $\varphi \rightarrow 0, \tilde{\mathcal{H}}_{\text {tun }}$ is a perturbative term and the linear response theory (see the Appendix) ensures that

$\mathcal{T}(\varepsilon)=\left(2 \pi V_{12}\right)^{2} \tilde{\rho}_{e}(\varepsilon) \tilde{\rho}_{o}(\varepsilon)$,

where $\tilde{\rho}_{e}(\varepsilon)=-\frac{1}{\pi} \operatorname{Im}\left(\tilde{\mathcal{G}}_{\Psi_{e} \Psi_{e}}\right)$ is the local density of states (LDOS) for the Hamiltonian of Eq. (4) and

$\mathcal{G}_{\Psi_{e} \Psi_{e}}=-\frac{i}{\hbar} \theta(\tau) \operatorname{Tr}\left\{\varrho_{\mathrm{e}}\left[\Psi_{e}(\tau), \Psi_{e}^{\dagger}(0)\right]_{+}\right\}$

gives the retarded Green's function in the time domain $\tau$, where $\theta(\tau)$ is the Heaviside step function, $\mathrm{Q}_{\mathrm{e}}$ is the density-matrix for Eq. (4), $\Psi_{e}=f_{e}+\left(\pi \Gamma \rho_{0}\right)^{1 / 2} q d_{1}$ is a field operator, with $f_{e}=\sum_{p} c_{e p}$, being the Anderson parameter $\Gamma=2 \pi V^{2} \rho_{0}$, with $\rho_{0}$ being the density of states for the STM tips and $q=\left(\pi \rho_{0} \Gamma\right)^{-1 / 2}\left(\frac{\sqrt{2} V}{2 V_{12}}\right)$.

To calculate Eq. (7) in the energy domain $\varepsilon$, we should employ the equation-of-motion (EOM) method [46] summarized as follows:

$\left(\varepsilon+i 0^{+}\right) \tilde{\mathcal{G}}_{\mathcal{A B}}=\left[\mathcal{A}, \mathcal{B}^{\dagger}\right]_{+}+\tilde{\mathcal{G}}\left[\mathcal{A}, \mathcal{H}_{i}\right] \mathcal{B}$

for the retarded Green's function $\tilde{\mathcal{G}}_{\mathcal{A B}}=\int d \tau \mathcal{G}_{\mathcal{A B}} e^{\frac{i}{\hbar}\left(\varepsilon+i 0^{+}\right) \tau}$, which is the time Fourier transform of $\mathcal{G}_{\mathcal{A B}}$, with $\mathcal{A}$ and $\mathcal{B}$ being fermionic operators belonging to the Hamiltonian $\mathcal{H}_{i}$. By considering $\mathcal{A}=\mathcal{B}=\Psi_{e}$ and $\mathcal{H}_{i}=\mathcal{H}_{e}$, we find

$\tilde{\mathcal{G}}_{\Psi_{e} \psi_{e}}=\tilde{\mathcal{G}}_{f_{e} f_{e}}+\left(\pi \rho_{0} \Gamma\right) q^{2} \tilde{\mathcal{G}}_{d_{1} d_{1}}+2\left(\pi \rho_{0} \Gamma\right)^{1 / 2} q \tilde{\mathcal{G}}_{d_{1} f_{e}}$.

From Eqs. (4) and (8) with $\mathcal{A}=\mathcal{B}=f_{e}$ and (9), we obtain 
$\tilde{\mathcal{G}}_{f_{e} f_{e}}=\frac{\pi \rho_{0}(\bar{q}-i)}{1-\sqrt{x}(\bar{q}-i)}+\pi \rho_{0} \Gamma\left[\frac{(\bar{q}-i)}{1-\sqrt{x}(\bar{q}-i)}\right]^{2} \tilde{\mathcal{G}}_{d_{1} d_{1}}$

and the mixed Green's function

$\tilde{\mathcal{G}}_{d_{1} f_{e}}=\sqrt{\pi \Gamma \rho_{0}} \frac{(\bar{q}-i)}{1-\sqrt{x}(\bar{q}-i)} \tilde{\mathcal{G}}_{d_{1} d_{1}}$,

determined from Eq. (8) by considering $\mathcal{A}=d_{1}, \mathcal{B}=f_{e}$ and $\mathcal{H}_{i}=\mathcal{H}_{e}$, with the parameter $x=\left(\pi \rho_{0} V_{12}\right)^{2}$ and $\bar{q}=\frac{1}{\pi \rho_{0}} \sum_{k} \frac{1}{\varepsilon-\varepsilon_{k}}$. Here we assume the wide band limit denoted by $\bar{q} \rightarrow 0$.

Additionally, for the Hamiltonian of Eq. (5) we have the LDOS $\tilde{\rho}_{o}(\varepsilon)=-\frac{1}{\pi} \operatorname{Im}\left(\tilde{\mathcal{G}}_{f_{0} f_{o}}\right)$, with

$\mathcal{G}_{f_{0} f_{o}}=-\frac{i}{\hbar} \theta(\tau) \operatorname{Tr}\left\{\mathrm{\varrho}_{0}\left[f_{o}(\tau), f_{o}^{\dagger}(0)\right]_{+}\right\}$

and $f_{o}=\sum_{\tilde{q}} c_{o \tilde{q}}$. We notice that $\tilde{\mathcal{G}}_{f_{o} f_{o}}$ is decoupled from the adatoms. Thereby, from Eqs. (5) and (12), we take $\mathcal{A}=\mathcal{B}=f_{o}$ and $\mathcal{H}_{i}=\mathcal{H}_{0}$ in Eq. (8) and we obtain

$\tilde{\mathcal{G}}_{f_{o} f_{o}}=\frac{\pi \rho_{0}(\bar{q}-i)}{1+\sqrt{x}(\bar{q}-i)}$.

Thus the substitution of Eqs. (9), (11), and (13) in Eq. (6), leads to

$\frac{\mathcal{T}(\varepsilon)}{\mathcal{T}_{b}}=1+\left(1-q_{b}^{2}\right) \tilde{\Gamma} \operatorname{Im}\left(\tilde{\mathcal{G}}_{d_{1} d_{1}}\right)+2 q_{b} \tilde{\Gamma} \operatorname{Re}\left(\tilde{\mathcal{G}}_{d_{1} d_{1}}\right)$

where $\tilde{\Gamma}=\frac{\Gamma}{1+x}$ is an effective adatom 1-tip coupling, $\mathcal{T}_{b}=\frac{4 x}{(1+x)^{2}}$ represents the transmittance through the STM tips when the adatom 1 is absent $(\tilde{\Gamma}=0), \mathcal{R}_{b}=1-\mathcal{T}_{b}$ stands for the corresponding reflectance and $q_{b}=\sqrt{\frac{\mathcal{R}_{b}}{\mathcal{T}_{b}}}=\frac{(1-x)}{2 \sqrt{x}}$ as the Fano parameter $[44,45,47]$. The current equation for the transmittance encodes three distinct Fano regimes of interference as follows: (i) $q_{b} \rightarrow \infty$ $(x=0)$ provides $\mathcal{T}(\varepsilon)=-\tilde{\Gamma} \operatorname{Im}\left(\tilde{\mathcal{G}}_{d_{1} d_{1}}\right)=\pi \tilde{\Gamma} \rho_{11}$ where we use $\rho_{11}=-\frac{1}{\pi} \operatorname{Im}\left(\tilde{\mathcal{G}}_{d_{1} d_{1}}\right)$ as the LDOS of the adatom 1 , which contains resonant states characterized by peaks, since the electronic transport in the interferometer is solely through the adatom 1 [15]; (ii) $q_{b}=0(x=1)$ leads to $\mathcal{T}(\varepsilon)=1-\pi \tilde{\Gamma} \rho_{11}$ exhibiting Fano dips as a result of the suppression caused by $\rho_{11}$ over the first term representing the maximum amplitude $\mathcal{T}_{b}=1$. In such a case, when $\rho_{11}$ shows a maximum, $\mathcal{T}(\varepsilon)$ presents a corresponding minimum as expected and the tunneling between the STM tips becomes the dominant process in the system. It reveals the depletion of charge in the LDOS of the STM tips detected by the transmittance, once this charge accumulates within the adatom 1, in particular, around the resonant states of $\rho_{11}$ observed in situation (i) for $q_{b} \rightarrow \infty$ $(x=0)$; (iii) corresponds to $q_{b} \approx 0.35(x=0.5)$, which is the intermediate case wherein asymmetric Fano lineshapes appear with peaks and Fano dips coexisting in the same profile, thus making explicit a competition between $V$ and $V_{12}$ on an equal footing. These features of Fano interference will be addressed in Section 3.

\subsection{System Green's functions}

By applying the EOM on

$\mathcal{G}_{d_{1} d_{1}}=-\frac{i}{\hbar} \theta(\tau) \operatorname{Tr}\left\{\varrho_{\mathrm{e}}\left[d_{1}(\tau), d_{1}^{\dagger}(0)\right]_{+}\right\}$,

and changing to the energy domain $\varepsilon$, we obtain the following relation:

$\left(\varepsilon-\varepsilon_{1}-\Sigma\right) \tilde{\mathcal{G}}_{d_{1} d_{1}}=1-t \tilde{\mathcal{G}}_{d_{2} d_{1}}-\Delta \tilde{\mathcal{G}} d_{2}^{\dagger} d_{1}$

expressed in terms of the self-energy $\Sigma=-(\sqrt{x}+i) \tilde{\Gamma}$ and Green's functions $\tilde{\mathcal{G}}_{d_{2} d_{1}}$ and $\tilde{\mathcal{G}}_{d_{2}^{\dagger} d_{1}}$. According to the EOM approach we find
$\tilde{\mathcal{G}}_{d_{2} d_{1}}=-\frac{t \tilde{\mathcal{G}}_{d_{1} d_{1}}}{\left(\varepsilon-\varepsilon_{2}+i 0^{+}\right)}+\frac{\Delta \tilde{\mathcal{G}}_{d_{1}^{\dagger} d_{1}}}{\left(\varepsilon-\varepsilon_{2}+i 0^{+}\right)}$

$\tilde{\mathcal{G}}_{d_{2}^{\dagger} d_{1}}=-\frac{\Delta \tilde{\mathcal{G}}_{d_{1} d_{1}}}{\left(\varepsilon+\varepsilon_{2}+i 0^{+}\right)}+\frac{t \tilde{\mathcal{G}}_{d_{1}^{\dagger} d_{1}}}{\left(\varepsilon+\varepsilon_{2}+i 0^{+}\right)}$

and

$\tilde{\mathcal{G}}_{d_{1}^{\dagger} d_{1}}=-2 t \Delta \tilde{K} \tilde{\mathcal{G}}_{d_{1} d_{1}}$

in which $\tilde{K}=\frac{K}{\varepsilon+\varepsilon_{1}+\bar{\Sigma}-K_{-}}$, with $K=\frac{\left(\varepsilon+i 0^{+}\right)}{\left[\varepsilon^{2}-\varepsilon_{2}^{2}+2 i \varepsilon 0^{+}-\left(0^{+}\right)^{2}\right]}, \bar{\Sigma}$ as the complex conjugate of $\Sigma$ and $K_{ \pm}=\frac{\left(\varepsilon+i 0^{+}\right)\left(t^{2}+\Delta^{2}\right) \pm \varepsilon_{2}\left(t^{2}-\Delta^{2}\right)}{\left[\varepsilon^{2}-\varepsilon_{2}^{2}+2 i \varepsilon 0^{+}-\left(0^{+}\right)^{2}\right]}$. Thus substituting Eqs. (17)-(19) into Eq. (16) Green's function of the adatom 1 becomes

$\tilde{\mathcal{G}}_{d_{1} d_{1}}=\frac{1}{\varepsilon-\varepsilon_{1}-\Sigma-\Sigma_{\mathrm{MFs}}}$,

where

$\Sigma_{\mathrm{MFs}}=K_{+}+(2 t \Delta)^{2} K \tilde{K}$

accounts for the self-energy due to the MFs connected to the adatom 1. We highlight that the self-energy of Eq. (21) contains the underlying mechanism that allows the invariance of the Fano lineshape. Such a universal feature is revealed as independent on the Fano parameter $q_{b}$, which we will discuss in detail below. Particularly for $t=\Delta=\frac{\lambda}{\sqrt{2}}$, we highlight that the expressions for $\tilde{K}$ and $\Sigma_{\mathrm{MFs}}$ found in Ref. [15] are recovered. Such a result will be revisited in Section 3.

\section{Results and discussion}

Below we investigate the features of the system Green's functions by employing the expression for the transmittance (Eq. (14)). According to Eq. (3), this transmittance can be obtained experimentally via the conductance $G$ in units of $G_{0}$ for temperatures $T \rightarrow 0$. Additionally, we employ values for the Fermi energy $\varepsilon, \varepsilon_{j}, t$, and $\Delta$ in units of the Anderson parameter $\Gamma$.

In Fig. 2 we consider the Fano regime $x=0 \quad\left(q_{b} \rightarrow \infty\right)$ for the transmittance $\mathcal{T}$ of Eq. (14) as a function of the Fermi energy $\varepsilon$. This situation corresponds to the case where the electron tunneling occurs exclusively through the adatom 1, due to the strong coupling between it and the STM tips. As predicted by the standard Fano's theory [44], the transmittance should exhibit a peak around each localized state in the adatom probed by the tips: see the green line shape of panel (a) for the adatom 1 here assumed to be decoupled from the adatom 2 for the sake of simplicity, which leads to the resonance centered at $\varepsilon=\varepsilon_{1}=-5$ with maximum amplitude $\mathcal{T}=1$. By keeping this level at such a value and employing $t=\Delta=4$ combined with $\varepsilon_{2}=0$, a ZBP given by $\mathcal{T}=1 / 2$ emerges due to the MF existing in adatom 1 [5]. Additionally, the most left resonance in the same curve corresponds to that at $\varepsilon=-5$ found in the green lineshape, in particular with renormalized peak position $\varepsilon \approx-10$ as the aftermath of the connection $\sqrt{2} \Delta$ with the adatom 2 , and with higher amplitude $(\mathcal{T}>1 / 2)$ in respect to that for the ZBP $(\mathcal{T}=1 / 2)$. Notice that a third peak in the vicinity of $\varepsilon \approx+10$ is found characterized by $\mathcal{T}<1 / 2$. Thus in the presence of finite couplings $t$, the original peak at $\varepsilon=\varepsilon_{1}=-5$ in the green curve of panel (a) with $\mathcal{T}=1$ is split into those at $\varepsilon \approx-10$ and $\varepsilon \approx+10$ both with $\mathcal{T}<1$ being the red lineshape points out. In which concerns the curves for $t<\Delta(t=2$ and $\Delta=4)$ and $t>\Delta(t=4$ and $\Delta=2)$, the transmittance is revealed as independent on the strengths $t$ and $\Delta$. Such a behavior attests 

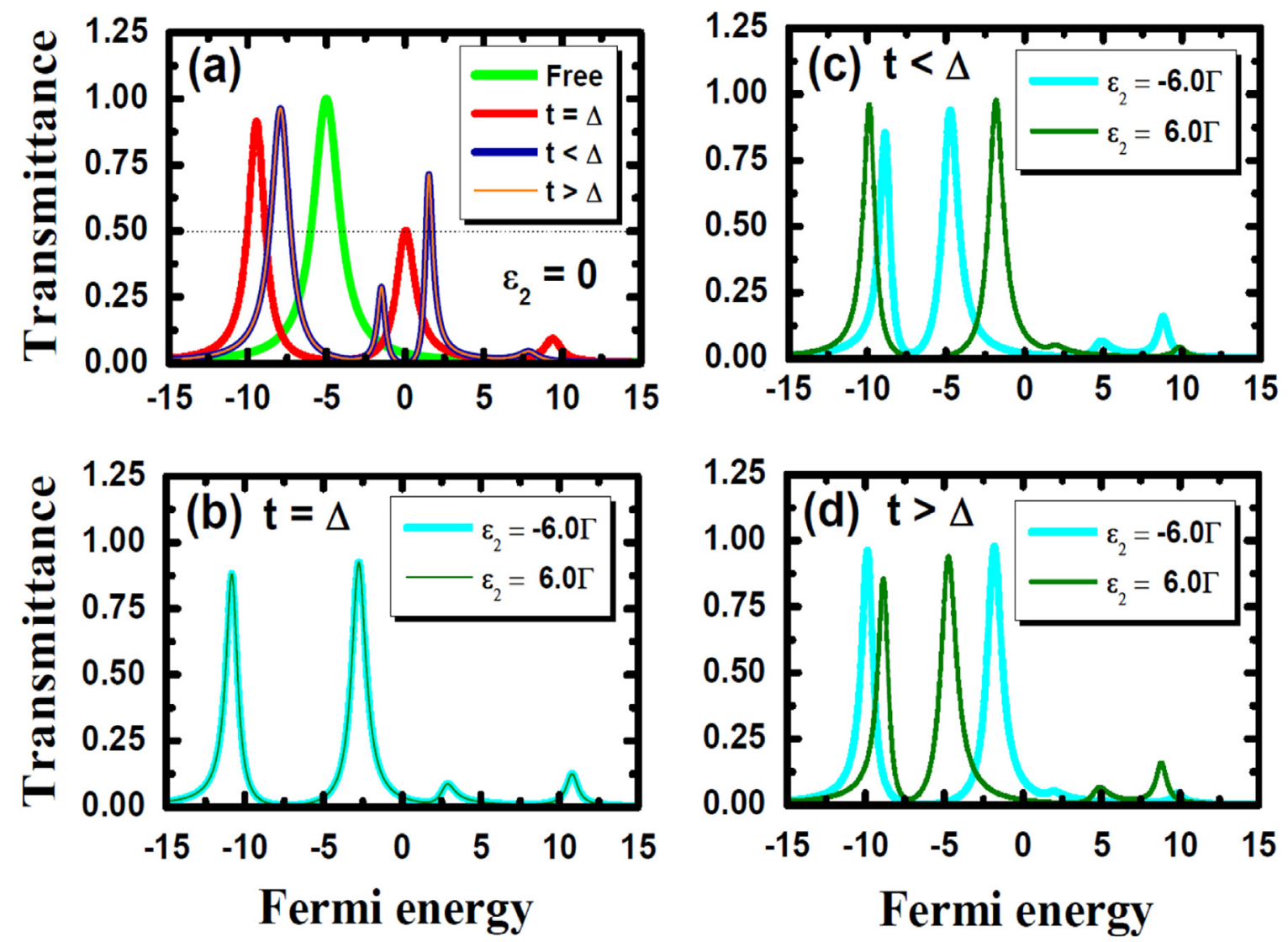

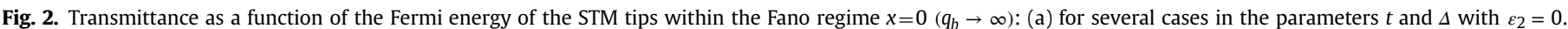

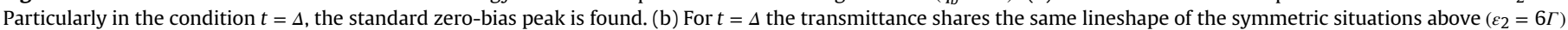

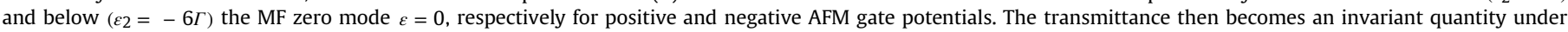

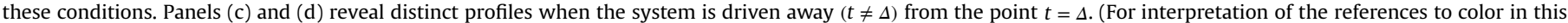
figure caption, the reader is referred to the web version of this paper.)

the situation in which coupled MFs are absent in the system within the adatom 2.

Panel (b) of Fig. 2 depicts the situation in which the system is still within the regime $t=\Delta$, but with $\varepsilon_{2} \neq 0$. We point out that such a panel reveals a universal behavior in the transmittance profile when symmetric values for $\varepsilon_{2}$ are accounted. This regime is characterized by coupled MFs (see Fig. 1(d)) which result in the suppression of the ZBP and the splitting of the resonances at $\varepsilon \approx-10$ and $\varepsilon \approx+10$ observed in the red curve of panel (a). For instance, the aforementioned universality is verified providing two identical curves for both values $\varepsilon_{2}=6 \Gamma$ (positive potential) and $\varepsilon_{2}=-6 \Gamma$ (negative potential) due to the self-energy $\Sigma_{\mathrm{MFs}}$ of Eq. (21) for the MFs, which is dependent on the amplitude $K_{ \pm}$. Notice that $K_{ \pm}=\frac{2 t^{2}\left(\varepsilon+i 0^{+}\right)}{\left[\varepsilon^{2}-\varepsilon_{2}^{2}+2 i \varepsilon 0^{+}-\left(0^{+}\right)^{2}\right]}$ within this situation, thus implying in $K_{ \pm}\left(\varepsilon_{2}\right)=K_{ \pm}\left(-\varepsilon_{2}\right)$ as well as $\Sigma_{\mathrm{MFs}}\left(\varepsilon_{2}\right)=\Sigma_{\mathrm{MFs}}\left(-\varepsilon_{2}\right)$, which then ensure the invariance of the transmittance profile at the point $t=\Delta$. For $t \neq \Delta$ we have $K_{ \pm} \propto \pm \varepsilon_{2}\left(t^{2}-\Delta^{2}\right)$, thus allowing a strong dependence on the sign of $\varepsilon_{2}$. In panels (c) and (d) of the same figure, the transmittance respectively for $t<\Delta(t=2$ and $\Delta=4)$ and $t>\Delta(t=4$ and $\Delta=2)$ exhibit distinct behaviors as expected when we adopt symmetric values $\varepsilon_{2}=6 \Gamma$ and $\varepsilon_{2}=-6 \Gamma$. Therefore in both limits $t>\Delta$ and $t<\Delta$, the influence of the negative potential $\varepsilon_{2}<0$ on the transmittance is made explicit once $\Sigma_{\mathrm{MFs}}\left(\varepsilon_{2}\right) \neq \Sigma_{\mathrm{MFs}}\left(-\varepsilon_{2}\right)$.

Fig. 3 holds within the Fano limit $x=1 \quad\left(q_{b}=0\right)$ where the electron tunneling between the STM tips is the dominant process in the system, thus resulting in Fano antiresonances instead of peaks in the transmittance profiles as a function of the Fermi energy. Panel (a) first displays the case in which the adatom 1 is decoupled from the adatom 2 represented by the green curve characterized by a dip at $\varepsilon=\varepsilon_{1}=-5$. By using finite values for $t$ and $\Delta$ combined with $\varepsilon_{2}=0$, we can observe the crossover from the regime $t \neq \Delta$ ( $t=2$ with $\Delta=4$ for the blue lineshape and $t=4$ with $\Delta=2$ in the case of the curve for the orange color) towards the point $t=\Delta=4$, where we can clearly realize in the red curve the emergence of a dip with amplitude $\mathcal{T}=1 / 2$, analogously to the opposite Fano regime of interference $\left(x=0\right.$ and $\left.q_{b} \rightarrow \infty\right)$ found in Fig. 2(a). In the presence of the potential $\varepsilon_{2} \neq 0$, the zero-bias dip disappears according to the curves with $\varepsilon_{2}=6 \Gamma$ (positive potential) and $\varepsilon_{2}=-6 \Gamma$ (negative potential) as found in panel (b) of the same figure. As in Fig. 2(b) we also report a universality feature in the transmittance profile, which still arises from the condition $\Sigma_{\mathrm{MFs}}\left(\varepsilon_{2}\right)=\Sigma_{\mathrm{MFs}}\left(-\varepsilon_{2}\right)$ apart from the Fano parameter as we can notice in Eq. (21). For $t \neq \Delta$ coincident curves no longer exist and the universal behavior is not verified as pointed out by panels (c) and (d), which have the same set of parameters as in Fig. 2.

To make explicit that the invariance feature of the gate potential for the point $t=\Delta=4$ is achievable for any Fano ratio $q_{b}$, we present in Fig. 4 the case $x=0.5\left(q_{b} \approx 0.35\right)$ in which both paths $V$ and $V_{12}$ of Eq. (1) compete on an equal footing. For this situation, we find intermediate Fano profiles where the underlying physics of Figs. 2 and 3 is still the same. Therefore based on the results of Figs. 2-4, we demonstrate that the invariance of the Fano profile is independent on $q_{b}$ arising solely from the feature $\Sigma_{\mathrm{MFs}}\left(\varepsilon_{2}\right)=\Sigma_{\mathrm{MFs}}\left(-\varepsilon_{2}\right)$, due to the connected MFs appearing in the term $i_{\varepsilon_{2}} \Psi_{A} \Psi_{B}$ within Eq. (2), in particular for $t=\Delta$. Moreover, the invariance with the potential $\varepsilon_{2}$ in the transmittance becomes clearer if we look to its density plot spanned by the axes $\varepsilon$ (Fermi 

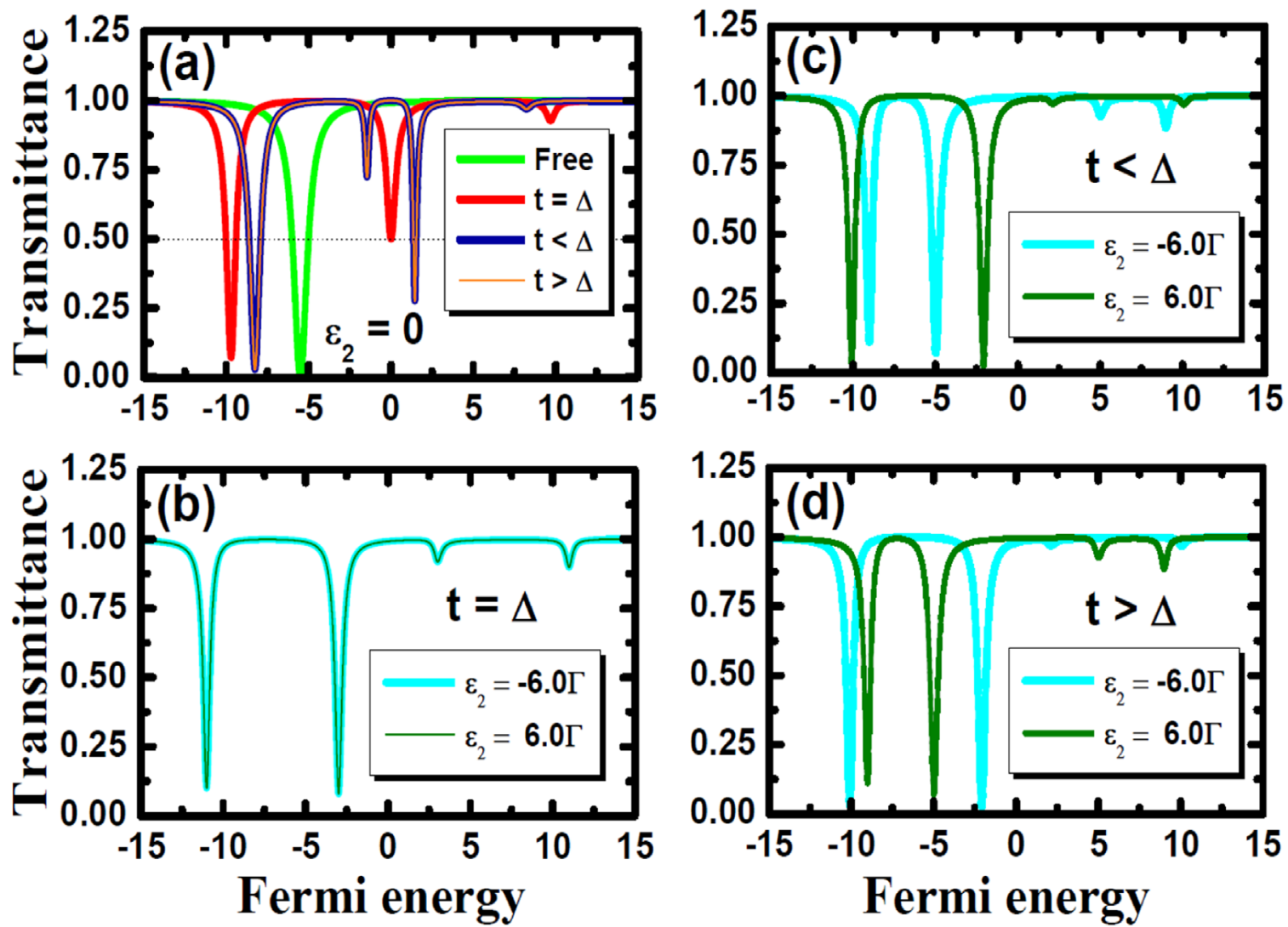

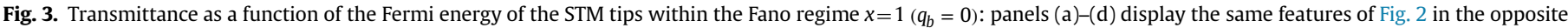
regime of interference. (For interpretation of the references to color in this figure caption, the reader is referred to the web version of this paper.)
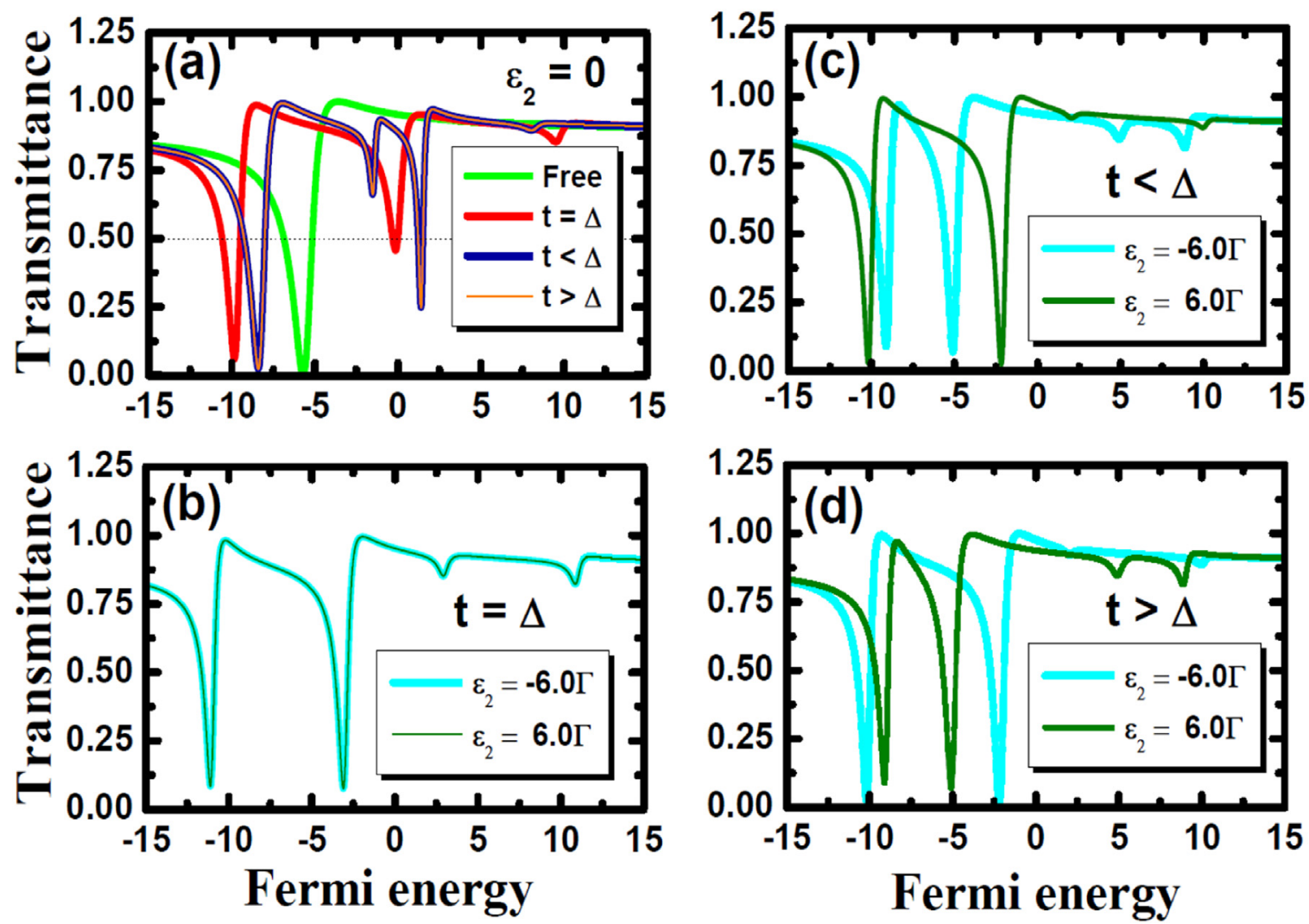

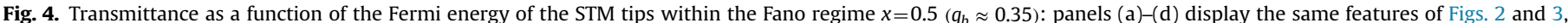
thus attesting that the point $t=\Delta$ is protected against the Fano effect as well as the symmetric change of the level in the adatom 2 . 

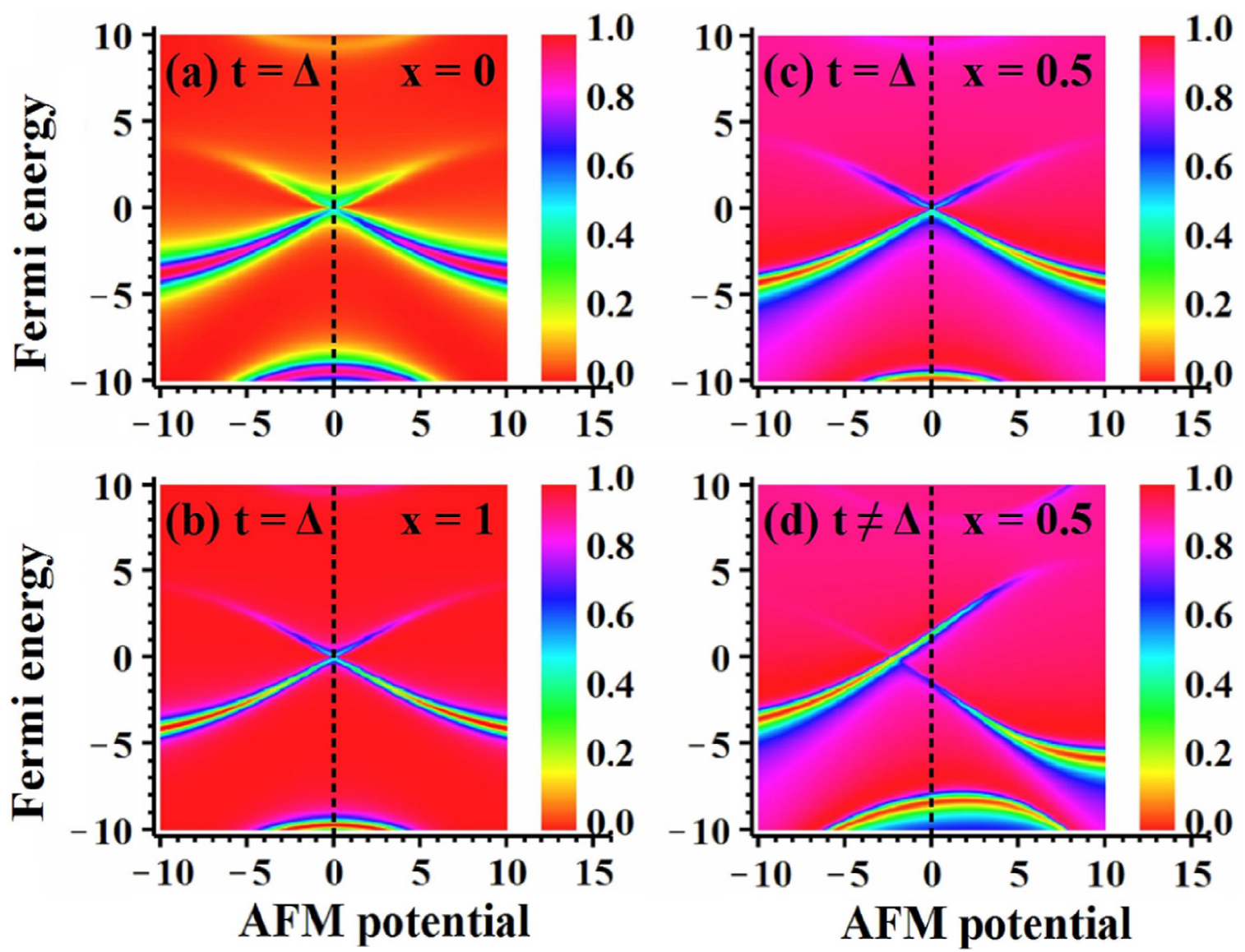

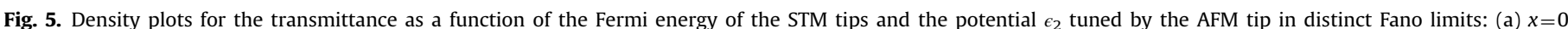

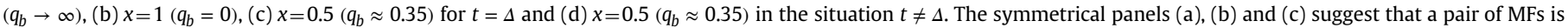

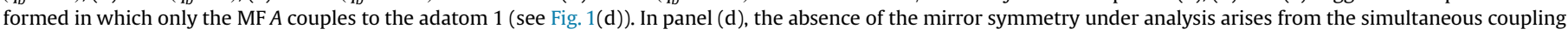

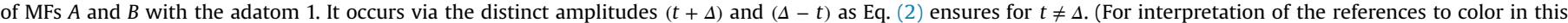
figure caption, the reader is referred to the web version of this paper.)

level) and $\varepsilon_{2}$. Fig. 5(a) is for $\varepsilon_{1}=-5$ and $t=\Delta=4$ : it exhibits the case $x=0 \quad\left(q_{b} \rightarrow \infty\right)$ for the regime of Fano interference, which shows the mirror symmetry under consideration with respect to the vertical axis placed at $\varepsilon_{2}=0$ (see the vertical dashed lines in the same figure). Notice that such a feature also manifests itself in panels (b) and (c), respectively in the limits $x=1$ and $x=0.5$. Here the orange color designates perfect insulating regions and those conducting are represented by red color. In panel (d) of the current figure, this mirror symmetry signature is broken just by using $t \neq \Delta$ as expected $(\Delta=2$ and $t=4)$.

The invariant Fano profiles for the transmittance found in panels (b) of Figs. 2-4 as well as those (a), (b) and (c) for Fig. 5 are due to the symmetric swap of the adatom 2 level around the MF zero mode which reveals the formation of a pair of MFs within such an adatom when $t=\Delta$. It couples the MF $A$ to the adatom 1 with amplitude $\sqrt{2} \Delta$ as Eq. (2) ensures. However in the regime $t \neq \Delta$, the pair of MFs still exists within the adatom 2 , but with the MFs $A$ and $B$ hybridized distinctly with the adatom 1 via the strengths $(t+\Delta)$ and $(\Delta-t)$, respectively. This feature is then probed by the symmetric tuning of the potential $\varepsilon_{2}$ around the MF zero mode, which yields the panels (c) and (d) of Figs. 2-4, in addition to the density plot in panel (d) of Fig. 5. Therefore, the aforementioned mechanism ruling the transmittance profiles via the couplings of the MFs with the adatom 1 is encoded by the selfenergy $\Sigma_{\mathrm{MFs}}$ of Eq. (21).

\section{Conclusions}

In summary, we have explored theoretically a Fano interferometer composed by STM and AFM tips over superconducting adatoms, in which the pair of MFs under the latter, elucidates the gate invariance feature of the interferometer, due to the Majorana nature arising from the adatom 2. Particularly for the situation where only one MF hybridizes with the adatom coupled to STM tips, the aforementioned invariance consists of a universal behavior within the transmittance when the AFM tip tunes symmetrically the energy level of its adatom around the MF zero mode. Such a universality is constituted by a common Fano profile in transmittance as a function of the Fermi level of the STM tips for two symmetric values for the AFM tip potential. In the case of two MFs connected to the adatom beneath the STM tips, we verify that such a universality is broken. Hence, despite the experimental challenging of the proposal, we expect that in the near future such an interferometer can be developed.

\section{Acknowledgments}

This work was supported by the Brazilian agencies CNPq, CAPES, 2014/14143-0 and 2015/23539-8 São Paulo Research Foundation (FAPESP). 


\section{Appendix A. Transmittance derivation}

Here we use the interaction picture to calculate $\mathcal{T}(\varepsilon)$ from Eq. (6). It ensures that a state $\left|\Phi_{n}\right\rangle$ from the spectrum of the Hamiltonian given by $\mathcal{H}_{e}+\mathcal{H}_{0}=\mathcal{H}_{\varphi=0}$ admits the following timedependency:

$\left|\Phi_{n}\right\rangle=e^{-\frac{i}{\hbar} \int_{-\infty}^{0} \tilde{\mathcal{H}} \operatorname{tun}(\tau) d \tau}\left|\Psi_{n}\right\rangle \simeq\left(1-\frac{i}{\hbar} \int_{-\infty}^{0} \tilde{\mathcal{H}}_{\operatorname{tun}}(\tau) d \tau\right)\left|\Psi_{n}\right\rangle$,

where $\hbar=\frac{h}{2 \pi}$ and $\left|\Psi_{n}\right\rangle$ is an eigenstate of $\mathcal{H}_{\varphi=0}$. Thus the current $\mathcal{J}_{\text {tip-1 }}$ for the STM tip 1 can be obtained by performing the expected mean value of the current operator $\mathcal{I}_{\text {tip-1 }} \equiv \mathcal{I}_{\text {tip-1 }}(t=0)$, which reads

$$
\begin{aligned}
\mathcal{J}_{\text {tip }-1}= & \left\langle\Phi_{n}\left|\mathcal{I}_{\text {tip }-1}\right| \Phi_{n}\right\rangle=-\frac{i}{\hbar}\left\langle\Psi_{n}\right| \int_{-\infty}^{0}\left[\mathcal{I}_{\text {tip }-1}, \tilde{\mathcal{H}}_{\text {tun }}(\tau)\right] d \tau \\
& \left|\Psi_{n}\right\rangle+O\left(\tilde{\mathcal{H}}_{\text {tun }}^{2}\right),
\end{aligned}
$$

where we have regarded $\left\langle\Psi_{n}\left|I_{\text {tip-1 }}\right| \Psi_{n}\right\rangle=0$ and by considering the thermal average on the latter equation, which gives

$\mathcal{J}_{\text {tip }-1}=-\frac{i}{\hbar} \int_{-\infty}^{0} \operatorname{Tr}\left\{\mathrm{\varrho}_{\varphi=0}\left[\mathcal{I}_{\text {tip }-1}, \tilde{\mathcal{H}}_{\text {tun }}(\tau)\right]\right\} d \tau$,

where $\mathrm{Q}_{\varphi=0}$ is the density matrix of the system described by the Hamiltonian $\mathcal{H}_{\varphi=0}$. By applying the equation-of-motion on $\mathcal{I}_{\text {tip- } 1 \text {, }}$ we show that

$$
\begin{aligned}
\mathcal{I}_{\text {tip }-1}= & -\frac{i}{\hbar}\left[e \sum_{k} c_{1 k}^{\dagger} c_{1 k}, \mathcal{H}_{\varphi=0}\right] \\
= & \left(-\frac{i e}{\sqrt{2} \hbar}\right) V \sum_{k}\left\{\left(c_{e k}^{\dagger} d_{1}-d_{1}^{\dagger} c_{e k}\right)+\left(c_{o k}^{\dagger} d_{1}-d_{1}^{\dagger} c_{o k}\right)\right\} \\
& +\left(-\frac{i e}{\hbar}\right) V_{12} \sum_{q \tilde{q}}\left(c_{o q}^{\dagger} c_{e \tilde{q}}-c_{e \tilde{q}}^{\dagger} c_{o q}\right),
\end{aligned}
$$

which, in combination with Eq. (A.3), leads to

$\mathcal{J}_{\text {tip }-1}=-\frac{e}{\hbar} \Delta \mu \operatorname{Im} \int_{-\infty}^{+\infty} d \tau\left\{\sqrt{2} V \mathcal{F}(-\tau)+2 V_{12} \mathcal{M}(-\tau)\right\}$,

where

$\mathcal{F}(-\tau)=-\frac{i}{\hbar} \theta(-\tau) \operatorname{Tr}\left\{\mathrm{e}_{\varphi=0}\left[f_{o}^{\dagger} d_{1}, \sum_{k} c_{e k}^{\dagger}(\tau) c_{o k}(\tau)\right]\right\}$

and

$\mathcal{M}(-\tau)=-\frac{i}{\hbar} \theta(-\tau) \operatorname{Tr}\left\{\mathrm{\varrho}_{\varphi=0}\left[f_{0}^{\dagger} f_{e}, \sum_{k} c_{e k}^{\dagger}(\tau) c_{o k}(\tau)\right]\right\}$

are retarded Green's functions.

In order to find a closed expression for the current $\mathcal{J}_{\text {tip-1 }}$, we should evaluate the integrals in the time coordinate $\tau$ of Eq. (A.5), which result in

$$
\begin{aligned}
\int_{-\infty}^{+\infty} d \tau \mathcal{F}(-\tau)= & \mathcal{Z}^{-1} \sum_{m n} \frac{\left(e^{-\beta E_{n}}-e^{-\beta E_{m}}\right)}{E_{n}-E_{m}+i 0^{+}} \times\left\langle\Psi_{n}\left|f_{o}^{\dagger} d_{1}\right| \Psi_{m}\right\rangle \\
& \times\left\langle\Psi_{m}\left|\sum_{k} c_{e k}^{\dagger} c_{o k}\right| \Psi_{n}\right\rangle
\end{aligned}
$$

and

$$
\begin{aligned}
\int_{-\infty}^{+\infty} d \tau \mathcal{M}(-\tau)= & \mathcal{Z}^{-1} \sum_{m n} \frac{\left(e^{-\beta E_{n}}-e^{-\beta E_{m}}\right)}{E_{n}-E_{m}+i 0^{+}} \times\left\langle\Psi_{n}\left|f_{o}^{\dagger} f_{e}\right| \Psi_{m}\right\rangle \\
& \times\left\langle\Psi_{m}\left|\sum_{k} c_{e k}^{\dagger} c_{o k}\right| \Psi_{n}\right\rangle,
\end{aligned}
$$

where we have used $\mathcal{Z}$ as the partition function of $\mathcal{H}_{\varphi=0}\left|\Psi_{m}\right\rangle=E_{m}\left|\Psi_{m}\right\rangle, \mathcal{A}(\tau)=e^{\frac{i}{\hbar}} \mathcal{H}_{\varphi=0^{\tau}} \mathcal{A} e^{-\frac{i}{\hbar} \mathcal{H}_{\varphi=0} \tau}$ for an arbitrary timedependent operator $\mathcal{A}(\tau)$. To eliminate the matrix element $\left\langle\Psi_{m}\left|c_{e k}^{\dagger} c_{o k}\right| \Psi_{n}\right\rangle$ in Eqs. (A.8) and (A.9), we calculate $\left\langle\Psi_{m}\left|\left[\sum_{k} c_{e k}^{\dagger} c_{o k}, \mathcal{H}_{\varphi=0}\right]\right| \Psi_{n}\right\rangle$, which gives

$$
\begin{aligned}
\left\langle\Psi_{m}\right. & \left.\left|\sum_{k} c_{e k}^{\dagger} c_{o k}\right| \Psi_{n}\right\rangle \\
& =\frac{-\sqrt{2} V\left\langle\Psi_{m}\left|d_{1}^{\dagger} f_{o}\right| \Psi_{n}\right\rangle}{\left(E_{n}-E_{m}\right)}-\frac{2 V_{12}\left\langle\Psi_{m}\left|f_{e}^{\dagger} f_{o}\right| \Psi_{n}\right\rangle}{\left(E_{n}-E_{m}\right)} .
\end{aligned}
$$

By performing the substitutions of Eqs. (A.8) and (A.9) with (A.10) in Eq. (A.5), we enclose the result into the function labeled by $\chi_{m n}$ to show that

$$
\begin{aligned}
\mathcal{J}_{\text {tip }-1} & =\frac{e}{\hbar} \pi \Delta \mu \mathcal{Z}^{-1} \sum_{m n} \chi_{m n} \frac{\left(e^{-\beta E_{n}}-e^{-\beta E_{m}}\right)}{E_{n}-E_{m}} \times \delta\left(E_{n}-E_{m}\right) \\
& =-\frac{e}{\hbar} \pi \Delta \mu \beta \sum_{m n}\left[\mathcal{Z}^{-1} e^{-\beta E_{n}} \delta\left(E_{n}-E_{m}\right)\right] \times \chi_{n m},
\end{aligned}
$$

where we have defined

$$
\begin{aligned}
\chi_{n m}= & (\sqrt{2} V)^{2}\left\langle\Psi_{n}\left|f_{o}^{\dagger} d_{1}\right| \Psi_{m}\right\rangle\left\langle\Psi_{m}\left|d_{1}^{\dagger} f_{o}\right| \Psi_{n}\right\rangle \\
& +2 \sqrt{2} V\left(2 V_{12}\right)\left\langle\Psi_{n}\left|f_{o}^{\dagger} d_{1}\right| \Psi_{m}\right\rangle\left\langle\Psi_{m}\left|f_{e}^{\dagger} f_{o}\right| \Psi_{n}\right\rangle \\
& +\left(2 V_{12}\right)^{2}\left\langle\Psi_{n}\left|f_{o}^{\dagger} f_{e}\right| \Psi_{m}\right\rangle\left\langle\Psi_{m}\left|f_{e}^{\dagger} f_{o}\right| \Psi_{n}\right\rangle .
\end{aligned}
$$

In this calculation we have used

$\left\langle\Psi_{n}\left|f_{o}^{\dagger} d_{1}\right| \Psi_{m}\right\rangle\left\langle\Psi_{m}\left|f_{e}^{\dagger} f_{o}\right| \Psi_{n}\right\rangle=\left\langle\Psi_{n}\left|f_{o}^{\dagger} f_{e}\right| \Psi_{m}\right\rangle\left\langle\Psi_{m}\left|d_{1}^{\dagger} f_{o}\right| \Psi_{n}\right\rangle$,

with

$\frac{\left(e^{-\beta E_{n}}-e^{-\beta E_{m}}\right)}{E_{n}-E_{m}}=-\beta e^{-\beta E_{n}}$

in the limit $E_{n} \rightarrow E_{m}$. The property $\left[\mathcal{H}_{e}, \mathcal{H}_{o}\right]=0$ ensures the partitions $E_{n}=E_{n}^{e}+E_{n}^{o}$ and $\mathcal{Z}=\mathcal{Z}_{e} \mathcal{Z}_{0}$ for the Hamiltonians $\mathcal{H}_{e}$ and $\mathcal{H}_{0}$, respectively in the brackets of Eq. (A.11), thus leading to

$$
\begin{aligned}
\mathcal{Z}^{-1} e^{-\beta E_{n}} \delta\left(E_{n}-E_{m}\right)= & \frac{1}{\beta} \mathcal{Z}_{e}^{-1} \mathcal{Z}_{o}^{-1} \int d \varepsilon\left(-\frac{\partial f_{F}}{\partial \varepsilon}\right) \\
& \times\left(e^{-\beta E_{n}^{e}}+e^{-\beta E_{m}^{e}}\right)\left(e^{-\beta E_{n}^{o}}+e^{-\beta E_{m}^{o}}\right) \times \delta(\varepsilon \\
& \left.+E_{n}^{e}-E_{m}^{e}\right) \delta\left(\varepsilon+E_{n}^{o}-E_{m}^{o}\right) .
\end{aligned}
$$

Therefore, we substitute Eqs. (A.12) and (A.14) in Eq. (A.11) to calculate $G=\frac{\partial}{\partial \varphi} \mathcal{J}_{\text {tip }-1}(\varphi=0)$. The comparison of such a result with Eq. (3) allows us to find Eq. (6). We also verify that $G=\frac{\partial}{\partial \varphi} \mathcal{J}_{\text {tip }-2}(\varphi=0)$ for the STM tip 2, which is agreement with Refs. [11,12] that show for the case with symmetric couplings $V$ absence of Andreev currents, i.e., $\frac{\partial}{\partial \varphi} \mathcal{J}_{\text {tip }-1}(\varphi=0) \neq \frac{\partial}{\partial \varphi} \mathcal{J}_{\text {tip }-2}(\varphi=0)$.

\section{References}

[1] J. Alicea, Rep. Prog. Phys. 75 (2012) 076501.

[2] S.R. Elliott, M. Franz, Rev. Mod. Phys. 87 (2015) 137.

[3] K. Flensberg, Phys. Rev. Lett. 106 (2012) 090503.

[4] M. Leijnse, K. Flensberg, Phys. Rev. Lett. 107 (2012) 210502.

[5] M. Leijnse, K. Flensberg, Phys. Rev. B 86 (2012) 134528.

[6] A.Y. Kitaev, Phys. Usp. 44 (2001) 131. 
[7] A.A. Zyuzin, D. Rainis, J. Klinovaja, D. Loss, Phys. Rev. Lett. 111 (2013) 056802.

[8] D. Rainis, J. Klinovaja, L. Trifunovic, D. Loss, Phys. Rev. B 87 (2013) 024515.

[9] A. Zazunov, P. Sodano, R. Egger, New J. Phys. 15 (2013) 035033.

[10] D. Roy, C.J. Bolech, N. Shah, Phys. Rev. B 86 (2012) 094503.

[11] Y. Cao, P. Wang, G. Xiong, M. Gong, X.-Q. Li, Phys. Rev. B 86 (2012) 115311.

[12] W.-J. Gong, S.-F. Zhang, Z.-C. Li, G. Yi, Y.S. Zheng, Phys. Rev. B 89 (2014) 245413.

[13] Y.-X. Li, Z.M. Bai, J. Appl. Phys. 114 (2013) 033703.

[14] N. Wang, S. Lv, Y. Li, J. Appl. Phys. 115 (2014) 083706.

[15] D.E. Liu, H.U. Baranger, Phys. Rev. B 84 (2011) 201308(R).

[16] E. Vernek, P.H. Penteado, A.C. Seridonio, J.C. Egues, Phys. Rev. B 89 (2014) 165314.

[17] D.E. Liu, M. Cheng, R.M. Lutchyn, Phys. Rev. B 91 (2015) 081405(R).

[18] M. Cheng, M. Becker, B. Bauer, R.M. Lutchyn, Phys. Rev. X 4 (2014) 031051.

[19] A.C. Seridonio, E.C. Siqueira, F.A. Dessotti, R.S. Machado, M. Yoshida, J. Appl. Phys. 115 (2014) 063706.

[20] F.A. Dessotti, L.S. Ricco, M. de Souza, F.M. Souza, A.C. Seridonio, J. Appl. Phys. 116 (2014) 173701.

[21] A. Ueda, T. Yokoyama, Phys. Rev. B 90 (2014) 081405(R).

[22] J.-J. Xia, S.-Q. Duan, W. Zhang, Nano. Res. Lett. 10 (2015) 223.

[23] E.J.H. Lee, X. Jiang, R. Aguado, G. Katsaros, C.M. Lieber, S. De Franceschi, Phys. Rev. Lett. 109 (2012) 186802.

[24] V. Mourik, K. Zuo, S.M. Frolov, S.R. Plissard, E.P.A.M. Bakkers, L. P. Kouwenhoven, Science 336 (2012) 1003.

[25] A. Das, Y. Ronen, Y. Most, Y. Oreg, M. Heiblum, H. Shtrikman, Nat. Phys. 8 (2012) 887.

[26] A.F. Otte, M. Ternes, K.V. Bergmann, S. Loth, H. Brune, C.P. Lutz, C. F. Hirjibehedin, A.J. Heinrich, Nat. Phys, 4 (2008) 847.

[27] V. Madhavan, W. Chen, T. Jamneala, F. Crommie, Phys. Rev. B 64 (2001) 165412.

[28] N. Knorr, M.A. Schneider, L. Diekhöner, P. Wahl, K. Kern, Phys. Rev. Lett. 88 (2002) 096804

[29] C.Y. Lin, A.H.C. Neto, B.A. Jones, Phys. Rev. Lett. 97 (2007) 156102.
[30] M. Ternes, A.J. Heinrich, W.D. Schneider, J. Phys.: Condens. Matter 21 (2009) 053001.

[31] S.N. Perge, I.K. Drozdov, B.A. Bernevig, Ali Yazdani, Phys. Rev. B 88 (2013) 020407(R).

[32] A. Heimes, P. Kotetes, G. Schön, Phys. Rev. B 90 (2014) 060507(R).

[33] J. Li, H. Chen, I.K. Drozdov, A. Yazdani, B.A. Bernevig, A.H. MacDonald, Phys. Rev. B 90 (2014) 235433.

[34] S.N. Perge, I.K. Drozdov, J. Li, H. Chen, S. Jeon, J. Seo, A.H. MacDonald, B. A. Bernevig, A. Yazdani, Science 346 (2014) 602.

[35] Y. Peng, F. Pientka, L.I. Glazman, F. von Oppen, Phys. Rev. Lett. 114 (2015) 106801.

[36] R. Pawlak, M. Kisiel, J. Klinovaja, T. Meier, S. Kawai, T. Glatzel, D. Loss, E. Meyer, arxiv:1505.06078v2, 2015.

[37] W.-H. Wu, K.-D. Zhu, Nanotechnology 26 (2015) 195501.

[38] V. Cherepanov, E. Zubkov, H. Junker, S. Korte, M. Blab, P. Coenen, B. Voigtländer, Rev. Sci. Instrum. 83 (2012) 033707.

[39] S. Qin, T-H. Kim, Z. Wang, A.-P. Li, Rev. Sci. Instrum. 83 (2012) 063704.

[40] A. Roychowdhury, M.A. Gubrud, R. Dana, J.R. Anderson, C.J. Lobb, F. C. Wellstood, M. Dreyer, Rev. Sci. Instrum. 85 (2014) 043706.

[41] H. Sellier, B. Hackens, M.G. Pala, F. Martins, S. Baltazar, X. Wallart, L. Desplanque, V. Bayot, S. Huant, Semicond. Sci. Technol. 26 (2011) 064008.

[42] M. Topinka, B. LeRoy, S. Shaw, E. Heller, R. Westervelt, K. Maranowski, A. Gossard, Science 289 (2000) 2323.

[43] A. Kleshchonok, G. Fleury, J.L. Pichard, Phys. Rev. B 91 (2015) 125416.

[44] U. Fano, Phys. Rev. 124 (1961) 1866.

[45] A.E. Miroshnichenko, S. Flach, Y.S. Kivshar, Rev. Mod. Phys. 82 (2010) 2257.

[46] H. Haug, A.P. Jauho, Quantum kinetics in transport and optics of semiconductors, in: Springer Series in Solid-State Sciences, vol. 123, Springer, New York, 1996.

[47] W. Hofstetter, J. König, H. Schoeller, Phys. Rev. Lett. 87 (2001) 156803. 